\section{Failure to replicate Dissociation between Antidiuretic and Vasopressor Activity in Rat Posterior Pituitaries in vitro}

WE have reported a dissociation between antidiuretic and vasopressor activities of vasopressin in the posterior lobe of rat pituitary in vitro ${ }^{1}$. Posterior pituitaries incubated in Krebs-Ringer bicarbonate glucose lost nearly all antidiuretic activity, although vasopressor activity was reduced only slightly. Antidiuretic activity seemed to have been released into the incubation medium, for there was far less vasopressor than antidiuretic activity in the medium. Thorn could not confirm this discrepancy in the release of the two activities in vitro using slightly different conditions ${ }^{2}$. We repeated the experiments (unpublished) in 1966 and observed the dissociation, although less marked than originally. When we repeated the work in 1968, we were unable to demonstrate the dissociation, although the experiments were apparently performed in the same conditions as before ${ }^{1}$. We thought that conditions might have been somewhat different after $2 \mathrm{yr}$, and decided to investigate some modifications of the conditions in vitro and in vivo.

Antidiuretic and vasopressor activity was determined as described earlier ${ }^{1}$. When posterior pituitaries were incubated in Krebs-Ringer bicarbonate glucose at $21^{\circ} \mathrm{C}$ or $40^{\circ} \mathrm{C}$ instead of $37^{\circ} \mathrm{C}$, or at $p \mathrm{H} 7 \cdot 1$ or 7.7 instead of $7 \cdot 4$, or in air instead of 95 per cent oxygen and 5 per cent carbon dioxide, there was no significant difference in antidiuretic and vasopressor activities in the incubated posterior pituitaries or in the medium. Similarly, neither the use of a modified Locke solution ${ }^{3}$, unchanged or modified by adding a high concentration of potassium, or a low concentration of sodium, nor the use of a calcium and magnesium-free medium ${ }^{4}$, induced dissociation in the two activities in vitro.

Changing the in vivo conditions had no effect on the ratio of antidiuretic to vasopressor activity of the posterior lobe or the medium. Neither anoxia induced by compressing the throat for 1 min nor stress caused by exposing the feet to electric shocks for $45 \mathrm{~s}$ revealed any dissociation in antidiuretic and vasopressor activity in pituitary or medium. Water deprivation for 1, 3 or 6 days, which led to a marked decline in the hormone content of the posterior lobe and its release in vitro, or water loading for 3 days also failed to alter the ratio of antidiuretic to vasopressor significantly from 1.

Thus we failed to observe again the dissociation ${ }^{1}$ in these two activities when posterior pituitaries are incubated in vitro. At the moment we cannot explain our original observation and wish to withdraw our statement that two principles are present in the posterior lobe in vitro, one with pressor and one with antidiuretic activity.

\section{E. VAN KaMmen}

D. DE WIED

Rudolf Magnus Institute for Pharmacology,

Medical Faculty,

University of Utrecht.

Received February 28; revised April 11, 1969.

' Van Kammen, E., and de Wied, D., Nature, 211, 93 (1966).

2 Thorn, N. A., Acta Endocrinol., 53, 644 (1966).

${ }^{3}$ Donglas, W. W., and Poisner, A. M., J. Physiol., 172, 1 (1964).

- Dicker, S. F., and Greenbaum, A. L., J. Physiol., 126, 116 (1954).

\section{Functional Occlusion in Tribosphenic Molars}

ATTEMPts have been made to deduce mandibular movements in primitive mammals from dried skulls and particularly the morphology and wear on the teeth ${ }^{1,2}$.
The work reported here shows that this is not possible. Until mandibular movements are precisely determined for appropriate living mammals, the interpretation of the structure and evaluation of the function of mammalian teeth is extremely difficult. Studies of living mammals are particularly relevant at present because the evolution of pre-Tertiary mammals can now be explained with greater confidence using the recently discovered early mammalian material from the Triassic, Jurassic and Cretaceous.

We have started to study mastication in primitive mammals using a specially designed cinefluorographic unit and Didelphis marsupialis as the first experimental animal. This technique has been used with rabbits ${ }^{3}$, rat $^{4}$ and the wallaby (W. D. L. Ride, personal communication), all highly specialized mammals. Here we briefly describe our equipment and outline the results; a full account will be published later.

The cinefluorographic unit consists of a $125 \mathrm{kV}$ rotating anode tube aligned with a $15 \mathrm{~cm}$ image intensifier. A $16 \mathrm{~mm}$ cine and a television camera are mounted on the image intensifier and can be run simultancously, allowing continuous monitoring of the animal's behaviour which can be selectively recorded on both cinefilm and videotape. The particular advantage of the apparatus compared with standard diagnostic equipment is the flexibility of the mounting: the tube and intensifier can be raised and lowered, tracked horizontally and rotated through $360^{\circ}$, simultaneously if necessary. Any natural changes in the position of the animal relative to the plane of the beam can be accommodated, so distortion is reduced.

Most mammals, including all with primitive dentitions, are anisognathic, so that chewing can only occur on one side of the mouth at any one time. In a chewing stroke the molars on the balancing side cannot come into contact. Mills ${ }^{1,2}$ has reviewed molar evolution in a series of primitivo mammals including primates, insectivores, some marsupials and some bats. Ho deduced that in all cases the chewing stroke commenced with the jaw depressed and lateral to the active side at initial tooth contact and that an upwards and medial movement followed until the protocones of the upper molars were locked into the talonid basins of the lower molars and the jaw was symmetrically placed about the midline. This he described as the "buccal phaso" and the terminal position of the jaw as the "enntric occlusion" or the "centrie". Mills said that in many mammals the "buccal phase" was followed by a "lingual phase" in which the jaw moved further medially to bring the molars of the hitherto inactive balancing side into functional occlusion. He suggested that during this second phase of movement the protocones of the upper molars act as a pestle in the mortar of the talonid basins. This implies that chewing in the animals Mills examined consists of a single stroke involving both sides of the jaw in sequence.

The cinefluorographic study has shown that all fecding activity in Didelphis is cyclical, rhythmic and repetitive and that chewing occurs on one side of the mouth only for up to thirty cycles, depending on the type of food, before active and balancing sides are changed. In the early stages of mastication, the food is crushed and punctured by the tips of the molar and premolar cusps without their coming into contact. This crushing type of mastication produces a distinct set of wear facets on the tips of the cusps. After a long period of crushing. the softened food is then cut by the shearing surfaces on the slopes of the cusps of the upper and lower molars; this produces a second group of striated wear facets. The chewing cycle in Didelphis consists of three strokes. First, a preparatory stroke in which the lower jaw swings upwards and laterally towards the active side until contact between tooth and food or tooth and tooth is achieved; second, a power stroke in which the lower jaw on the active side continues to move upwards but also medially and finally anteriorly to terminate with the protocones locked in the talonid basins or an equivalent 